\title{
SOME ATTEMPTS AT CHEMOTHERAPY OF CANCER
}

\author{
W. Davis, Ph.D., A.R.C.S. \\ Research Chemist, Institute of Cancer Research, University of London
}

The development of anything like a systematic approach to the chemotherapy of cancer began with the discovery that some chemical substances were capable of so profoundly interfering with the processes of cell division that either mitosis was prevented or else the daughter cells from the mitotic division were so damaged that they died. It was apparently because of this property that these substances were capable of inhibiting the growth at least of experimental tumours, and in the most favourable cases, holding out some therapeutic advantage in the treatment of some or other of the lymphadenopathies.

Thus colchicine, a drug derived from the autumr crocus-or a more recently discovered derivative, deacetylmethylcolchicine (Demecolcin) -can inhibit the formation of the spindle in metaphase and so arrest mitosis. Demecolcin, which is much less toxic than the parent substance, has been reported to give good results in the treatment of chronic myeloid leukaemia. Other plant materials which possess similar antimitotic activity, podophyllin for example, have been found to inhibit experimental tumours but to offer no clinical advantage.

But it was the wartime studies of the physiological effects of the war gases, sulphur and nitrogen mustard, that greatly accelerated research into the possibility of chemically inhibiting abnormal growth. It had been observed that methyl di-2chloroethylamine (Fig. I) - the war gas known as

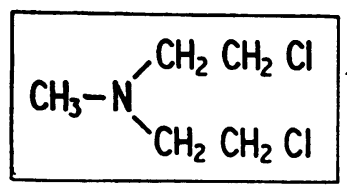

FIG. I

$\mathrm{HN}_{2}$ or ' nitrogen mustard'- - had a markedly destructive effect on the bone marrow and particularly on the haemopoietic system and it was soon being examined as a potential drug for the treatment of leukaemia, Hodgkin's disease and the lymphadenopathies in general. This drug has now been used over a period of more than ten years and is still considered a useful clinical tool. $\vec{\omega}$ Its dual character as a war gas, however, meant $\frac{}{\circ}$ that it was a most difficult drug to handle demanding careful intravenous injection to avoid vesication and producing often violent nausea.

The prime object of developments in this field was to produce a drug of similar potency but freed $i$ from the undesirable side-effects. One of the $f$ earliest examples in this direction was the di-2- 0 chloroethyl derivative of $\beta$-naphthylamine (Fig. 2)

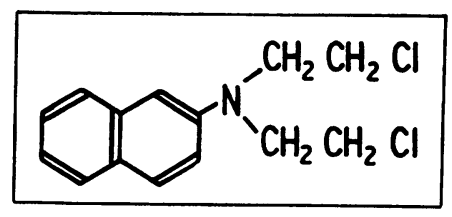

FIG. 2

which underwent extensive clinical trial under the name of R.48 (or Erysan). A vast number of drugs in this series have been synthesized by now and the majority of them are capable of inhibiting experimental tumours like the Walker rat carcinoma, of producing chromosome aberrations in dividing cells and mutations in Drosophila melanogaster - the fruit fly, but few of them display any specially desirable characteristics that would make them worthy of clinical investigation. However, in the search for some sort of specificity there have been just a few which do appear to offer some advantage over earlier members of the chloroethylamine - or nitrogen mustard-series.

I would like to make a brief diversion here to explain the rationale in so far as there is one, for $\frac{7}{0}$ the search for specificity. All the active nitrogen mustards are capable of inhibiting the growth of $N$ dividing cells, both normal and abnormal, in any $N$ part of the organism that they can reach in intact form. The intestinal mucosa and the bone marrow are thus particularly sensitive to attack. This implies that the search for specificity must

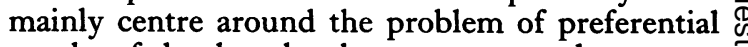
uptake of the drug by the tumour or at least more favourable transport of the drug to the site of action. To achieve this, compounds have been 
made in which the chloroethylamine group is linked to molecules which possess acidic, basic or amphoteric groups-possessing both basic and acidic characteristics. All these changes in the chemical nature of the compounds, also change their physical properties, increasing, for example, their water solubility. Within this range of drugs falls the derivative of $p$-aminophenylbutyric acid

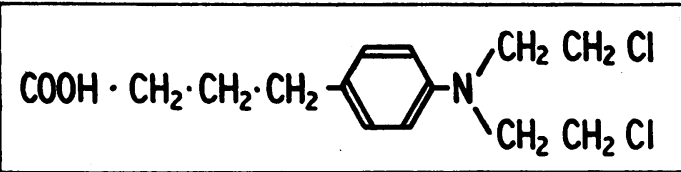

FIG. 3

known formerly as CB. 1348 (Fig. 3) and now being marketed as Chlorambucil.

Many clinicians here, in Europe and U.S.A. consider this the drug of choice in the treatment of chronic lymphocytic leukaemia, Hodgkin's disease and allied lymphomas. The drug probably owes its efficiency to the combination of a watersolubilizing carboxyl group in a moderate sized organic molecule, which results in a surface-active compound that could be transported without reacting in fatty droplets and then with equal ease transfer to aqueous medium in order to react in the cell. The amphoteric compound, di-2chloroethyl- $p$-aminophenylalanine (Fig. 4)-known

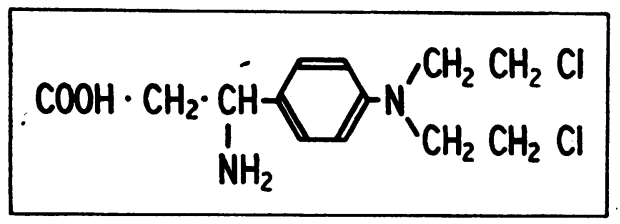

FIG. 4

as Melphalan in the $L$-form or Sarcolysine in the $D L$-form-is also undergoing extensive clinical investigation here and in the Soviet Union, where it was independently developed. This compound may depend for its apparent advantage on the uptake of the drug into the normal cell metabolism because it is derived from a naturally-occurring amino acid or it could be just another example of more favoured transport since homologous amino acid derivatives no longer occurring in nature apparently possess similar activity. There are other examples of nitrogen mustard derivatives which combine the active chloroethylamine group in a molecule closely related to naturally occurring substances; the pyridoxine derivative made in the U.S.A. (Fig. 5), Dopan (Fig. 6) a derivative of a pyrimidine, made in the Soviet Union and more recently BCM (Fig. 7) made by a group of Hungarian workers following this same trend, all appear to possess some anti-leukaemic activity.

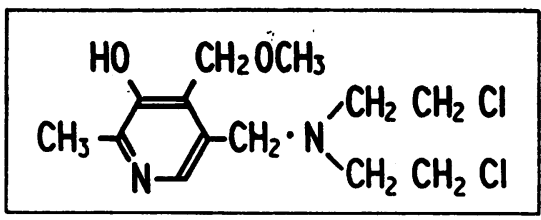

FIG. 5

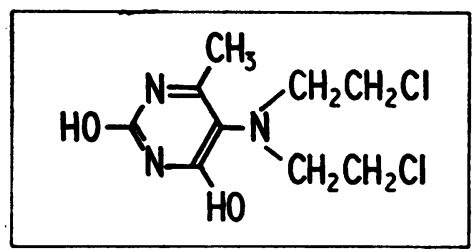

FIG. 6

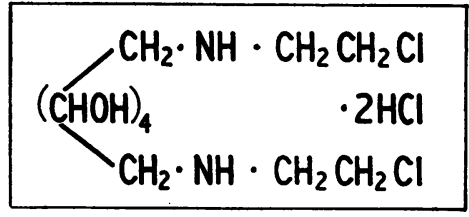

FIG. 7

It should be stressed, however, that the therapeutic advantage of any of these many derivatives over the parent $\mathrm{HN} 2$ depends almost entirely on ease of administration and freedom from un desirable side-effects.

As a series, the nitrogen mustards have a speciaf interest in the field of chemotherapy, for broadly speaking there is an observable correlation between chemical reactivity manifested by the ease with which the chlorine atoms ionize in aqueous medium and the biological activity, which can be expressed experimentally by the efficiency of the drug as an inhibitor of experimental tumours or by observable cytotoxic or mutagenic activity. This correlation is in fact the-starting point for the various theories of the mode of action of these drugs, which are considered to produce their effects by chemical reaction-or alkylation to be precise-of sorne of the chemically reactive groups present in the living cell. These include phosphoryl and basic groups in DNA, carboxyl, amino and sulphydryl group 3 .in protein. It is this ability to undergo the alkylating reaction under physiological conditions that is the common feature which links a number of other growth inhibiting drugs vith the nitrogen mustards. The methanesulphonyl esters, the ethyleneimines and the epoxides are: all growth inhibiting compounds and, like the nitrogen mustards, all alkylating agents.

Myleran, now well known in the treatment of chronic myeloid leukaemia, is di-methanesulphonoxybutane (Fig. 8). It owes its activity to the ease with which it eliminates the methanesulphonyl 


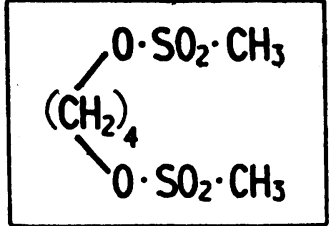

Fig. 8

group, comparable in some ways to the elimination of the chlorine atom from the nitrogen mustards. Other members of this series possess growth inhibiting action, but Myleran remains outstandingly active in this group.

The ethyleneimines, which can alkylate because of the ease with which the three-membered ring opens, are powerful growth inhibitors, and several drugs which are ethyleneimine derivatives are in use clinically. The most important member of

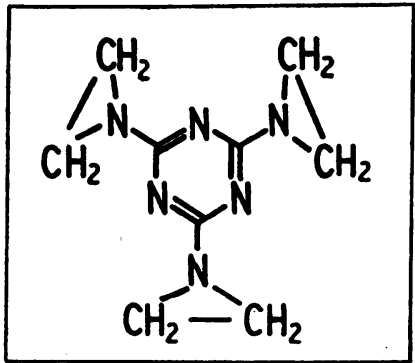

FIG. 9

this group is triethylenemelamine (TEM) (Fig. 9) which has been in use for seven years in the treatment of Hodgkin's disease, lymphosarcoma and the chronic leukaemias. Triethylenephosphoramide (TEPA) (Fig. Io) and triethylenethiophosphoramide (Thio-TEPA) (Fig. ro with ' $S$ ' in-

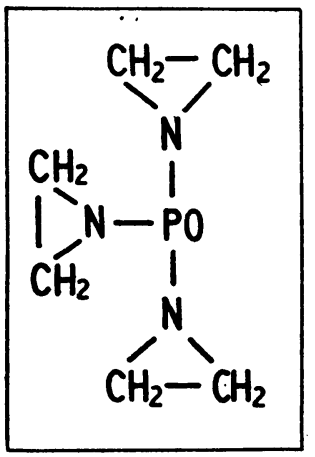

Fig. 10

stead of ' $O$ ') have received close attention in the past five years and they have been reported to have produced significant improvement in patients suffering from Hodgkin's disease, neuroblastoma and malignant melanoma. The important tem- porary effects on malignant melanoma, ovarian carcinoma and seminoma metastases achieved by thio-TEPA in particular certainly merit further attention.

In Germany, clinicians have recently reported results obtained in the treatment of a large number of patients with ethyleneiminobenzoquinone and its derivative (Fig. II) known as Bayer E 39 and

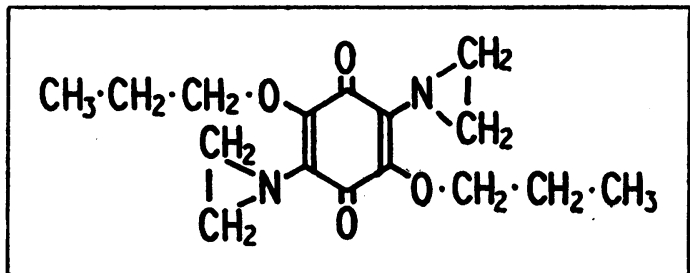

FIG. I I

A 139. They have concluded that in the treatment of chronic myeloid leukaemia, these drugs are comparable in their effectiveness with Myleran, and in the treatment of lymphatic leukaemia, with TEM.

Although the epoxides (Fig. 12) have been

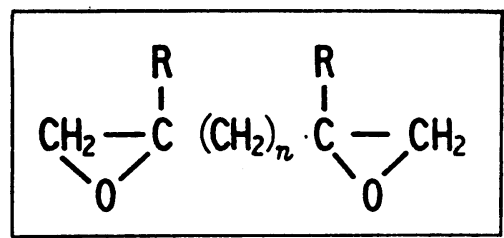

FIG. 12

demonstrated experimentally as growth inhibitors of the same type as the other alkylating agents, they have not shown any special advantage to justify clinical trials. Their main application has been in the hands of cytologists who have studied in detail the chromosomal abnormalities that follow treatment with these drugs. Comparative mutagenic studies have also been made by studying the effects of various alkylating agents on Drosophila melanogaster. Because of the observed similarities between the chromosomal aberrations produced by these drugs and by $\mathrm{X}$-rays and also because of their comparable mutagenic activity, the alkylating agents have been termed ' radiomimetic.'

The attempted therapy of the lymphadenopathies has not been limited to the alkylating agents. Urethane (Fig. 13) for example has been studied and used clinically to produce temporary

$$
\mathrm{C}_{2} \mathrm{H}_{5} \mathrm{O} \cdot \mathrm{CO} \cdot \mathrm{NH}_{2}
$$

FIG. 13 
remissions in cases of myelogenous leukaemia. Its mode of action is not certain but it has been suggested that its effect is achieved through interference in the incorporation of formate carbon into nucleic acids.

Because the synthesis of nucleic acid is so intimately concerned in the process of growth and cell division, the possibility of interfering with or inhibiting its synthesis has received a great deal of attention and from a long programme of research into potential antimetabolites, certain compounds that are similar to, but significantly different from, the naturally occurring bases of nucleic acid have been found to be biologically active and capable of producing some response when tested against leukaemia. Compounds have been synthesized that are purine antagonists; others, as folic acid antagonists. In the first category, 6-mercaptopurine (Fig. 14) (Purinethol), the most widely

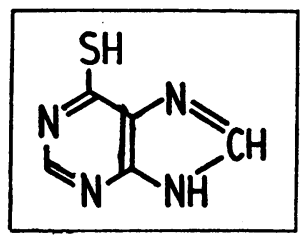

Frg. 14

known antimetabolite, is considered by some to be the drug of choice in the treatment of acute leukaemia in adults.

Other compounds in this field-and there have been very many derivatives synthesized-possess some therapeutic activity. They include thioguanine which has similar action to, and no advantage over, 6-mercaptopurine, 2 : 6-diaminopurine, and 6-chloropurine which both produced temporary remission in acute and chronic myeloid leukaemia, and 8-azaguanine (Guanazolo) which has been tested against Hodgkin's disease, lymphosarcoma and acute leukaemia and is apparently capable of producing some relief of symptoms but offers no advantage over the other antipurines.

A number of drugs that have been synthesized specifically as folic acid antagonists have been studied for their effects against leukaemia. 4-Aminopteroylglutamic acid (Aminopterin) is capable of producing some improvement in acute leukaemia in children, and with its related folic acid antagonist, Amethopterin (Methotrexate) is reported to produce impressive changes in patients with rhabdomyosarcoma, Hodgkin's disease, lymphosarcoma, neuroblastoma and chronic lymphoid leukaemia.

There is one example of an amino acid antagonist, $D L$-ethionine, an analogue of methionine, which is capable of inhibiting experimental tumours but clinical applications have been without value.

Very recently a new class of tumour inhibitory compounds has been synthesized, fluorinated pyrimidines. So far only experimental studies have been reported but two compounds, 5fluorouracil (FU) and 5-fluoro-orotic acid (FO) are effective inhibitors of experimental tumours and leukaemia.

A number of antibiotics derived from Streptomyces have been found to inhibit the growth of experimental tumours and in the case of at least one of them, azaserine (Fig. 15), this may be due

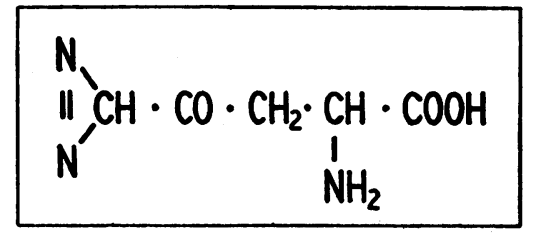

FIG. i 5

to its inhibitory action on purine synthesis, possibly by acting as an antagonist to $L$-glutamine, a metabolic precursor. The clinical trials with this new drug proved disappointing but a more active homologue, diazo-oxo- $L$-norleucine (DON) is now being studied.

Finally, adrenocorticotrophic hormone (ACTH) and cortisone, which play an important part in the treatment of acute leukaemias and some? others of the lymphoma group should be mentioned, though they should not be classed as chemotherapeutic agents in the same way as the other drugs for they are not growth inhibitors. Treatment with these drugs does not lend itself to maintenance therapy but in combination with an antimetabolite like 6-mercaptopurine they have been found very valuable in treating acute leukaemia.

It emerges clearly now that even though the range of substances that possess growth inhibitory activity is very wide, the type of effect they produce is limited to achieving at best important remissions in some of the lymphadenopathies. The solid tumours, both primary and secondary, remain an unanswered challenge for all who work in this field.

\section{BIBLIOGRAPHY}

HEIDELBERGER $C$ et al. (1957), Nature, 79063 CLARKE D. A., REILLLY, H. C., and CHESTER STOCK, C (1956), Proc. Amer. Assoc. Cancer Res., 2, 100. REVIEW ARTICLES

PHILIPS, F. S. (1950), 7. Pharmacol., 99, 28 I. HADDOW, A. (1953), 'Physiopathology of Cancer,' p. 475. New York.

ROSS, W. C. J. (1953), Adv. Cancer Res., i, 397, New York.

CHESTER STOCK, C. (1954), Ibid., ii, 426, New York.

FARBER, S., et al. (1956), Ibid., iv, 2, New York.

TIMMIS, G. M. (1957), Ұ. Pharm. Pharmacol., o, 81 .

'Comparative Clinical and Biological Effects of Alkylating Agents (1957), Ann. N.Y. Acad. Sci. (in press). 